\title{
Determining the Drying Out of Coniferous Trees Using Airborne and Satellite Data
}

\author{
Sviatlana I. Guliaeva, Ilya I. Bruchkousky, Leonid V. Katkovsky \\ Department of Aerospace Research, A.N. Sevchenko Research Institute of Applied Physical Problems of the Belarusian State \\ University, Minsk, Republic of Belarus \\ Email: ms.bruchkovskaya@yandex.ru
}

How to cite this paper: Guliaeva, S.I., Bruchkousky, I.I. and Katkovsky, L.V. (2021) Determining the Drying Out of Coniferous Trees Using Airborne and Satellite Data. Advances in Remote Sensing, 10, 25-46. https://doi.org/10.4236/ars.2021.102002

Received: March 25, 2021

Accepted: May 31, 2021

Published: June 3, 2021

Copyright $\odot 2021$ by author(s) and Scientific Research Publishing Inc. This work is licensed under the Creative Commons Attribution International License (CC BY 4.0).

http://creativecommons.org/licenses/by/4.0/ (c) (i) Open Access

\begin{abstract}
In recent decades, the problem of drying out of conifers has become a subject of significant importance due to the widespread mortality of trees caused by stem pest's damage. Early detection of areas affected by insect outbreaks is of great relevance for preventing the further spread of pests. Forests of Belarus are largely affected by conifers dieback caused by the bark beetle. The aim of the study was to identify drying out conifers using a TripleSat satellite multispectral image of a woodland area in Belarus based on preliminary airborne measurements. Spectrometers operating in a spectral range of $400-900 \mathrm{~nm}$ were used in airborne measurements, resulting in distinguishing various drying out stages with an accuracy of $27 \%-74 \%$ for aerial data. In this study, a supervised classification of the TripleSat image based on the method of linear discriminant analysis (LDA) was performed. The input data for LDA algorithm is a set of remote sensing vegetation indices. Results of the study demonstrate that about $90 \%$ of the test site is at the green-attack stage that is confirmed by ground surveys of this area.
\end{abstract}

\section{Keywords}

Remote Sensing, Forest Monitoring, Stem Pest, Spruce Dieback, Spectral Classification, Vegetation Indices, Belarus

\section{Introduction}

A noticeable deterioration of the state of coniferous forests is currently one of the most important problems of forestry in Belarus, many countries of Europe and North America [1] [2] [3]. The main predisposing factor causing conifers dieback is extreme climatic conditions-hurricanes [4] [5] and episodes of drought [6] [7] [8] [9] that provide necessary conditions for spreading of pests 
and pathogens over new areas. Meanwhile, conifers dieback constitutes significant damage to forest ecosystem and has a negative economic impact.

As a result of dry weather established in Belarus in last decades, decline and dieback of the spruce (Picea abies L.) formation has begun [2] and is still continuing. Colonization of weakened trees by the bark beetle (Ips typographus) [2] [10] was the major reason of spruce dieback in Belarus in 2019 [1] [11], being also the cause of drying out of spruce in $75 \%$ of cases in the forests of Central and Eastern Europe [12] [13]. Therefore, prompt monitoring of forest areas employing remote sensing methods is of interest in order to identify dying coniferous trees at the initial stage for follow-up activities concerning preventing and mitigation of dieback [14].

There are several stages of drying out of conifers [15]. The initial stage of conifer damage by a bark beetle is the so-called green-attack stage, while the crown visually looks healthy, although by the time of attack, the tree is already weakened. Red-attack stage begins, when the crown becomes thinner and the needles change their color to yellow or red-brown [16]. The gray-attack stage is the following, the needles fall off completely and the tree dries up.

Most of the studies focused on the determining both green-attack stage and characteristic parameters of conifers (tree height, features of the crown structure, amount of biomass, leaf surface index, moisture content) use satellite imagery [17] [18] [19] [20] as well as lidar and radar data sets [21] [22]; researches on the temporal variations in the parameters of coniferous forests [17] [18] are conducted so far. In addition to this, RGB- [18] or RGBNIR-bands [23] [24] airborne images are used as a priori data and Landsat, Sentinel-2, WorldView-2, IKONOS imagery are used as satellite data. The difficulty in green-attack stage detecting using broadband satellite sensors consists in significant similarity of the spectral responses of infected trees at an early stage and the uninfected ones [15], even in high spectral resolution data, and also in the difficulty of taking into account the role of optical properties of an undergrowth.

Until now, TripleSat sensor data has been used for crop monitoring and land cover analysis [25] [26] and has not been used for green-attack stage detection. In addition, the possibility of using classical wide range spectrometer's data as a source of more detailed spectral information to improve the quality of detection of the green-attack stage is relevant, due to the lack of studies focused on methods for determining the stages of drying out of conifers using such spectrometers.

In view of the foregoing, the aim of the study was to determine the green-attack stage detection potential of 4-band RGBNIR very high resolution $(0.8 \mathrm{~m})$ TripleSat data in combination with a priori airborne spectrometer's data in the range of $400-900 \mathrm{~nm}$. The assumption is made that this approach will minimize the contribution of undergrowth to the resulting reflection spectrum due to careful selection of individual conifers, while recording a continuous spectrum makes it possible to separate the features inherent to the green-attack stage. Information provided by foresters about stages of conifers drying has been used as an addi- 
tional reference data.

For the first time for the territory of Belarus (Novosady, Minsk region) estimates of the green-attack stage for a test forest area were made using airborne measurements. The estimates were verified using high resolution aerial images of the test area. Results of the study demonstrate the deplorable state of the test site (about $90 \%$ at the green-attack stage) and it is well consistent with the forester's expertise that there are almost no healthy trees in the study area. More accurate evaluation of the accuracy of the applied method, however, is difficult due to insufficient data on the state of the trees of the test site. High quality imagery of forest area under study and detailed ground truth data of forest pathological services should be used to access the classification accuracy.

\section{Materials}

\subsection{Study Area}

Forest site near the village of Novosady, Pukhovichi district, Minsk region $\left(53^{\circ} 39^{\prime} 15^{\prime \prime} \mathrm{N}, 27^{\circ} 38^{\prime} 25^{\prime \prime} \mathrm{E}\right)$ covering $0.1 \mathrm{~km}^{2}$ was chosen as a test area for the research (Figure 1). The site contains mainly spruce with a slight inclusion of deciduous trees. The selection of the site was carried out jointly with the specialists of the Kaikovsky forestry of the General Directorate of Forestry "Minsk Forestry" taking into account a priori data on the state of health of forest.

The site belongs to the subzone of broadleaf-spruce (oak-dark coniferous) forests. The climate of the region is moderately continental. The height of the site above sea level is about $160 \mathrm{~m}$. The average annual air temperature is $+6.1^{\circ} \mathrm{C}$. The amount of precipitation per year is $676 \mathrm{~mm}$, with an average amount per month of $44 \mathrm{~mm}$ in winter and $82 \mathrm{~mm}$ in summer. The average temperature in winter is $-6^{\circ} \mathrm{C}$, in summer $\pm 22^{\circ} \mathrm{C}$.

In 2018 the forest area generally consisted of middle-aged spruce, according to the vegetation maps of Minsk forestry. By the time of the study, which was carried out on the 10th of July 2020, the central part of the site had been subjected to sanitary cutting due to bark beetle infestation. When examining the remaining

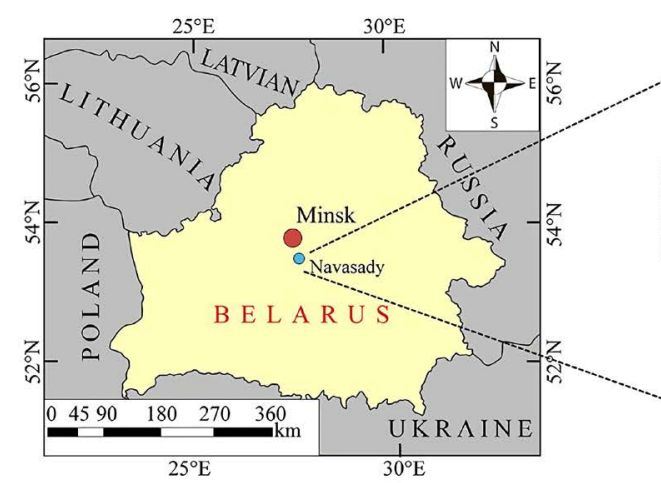

(a)

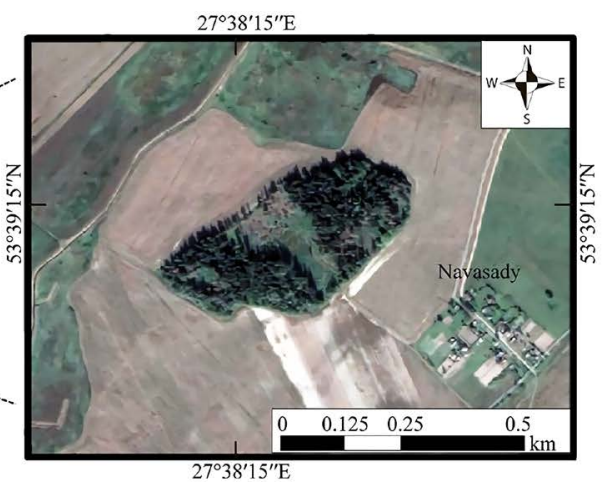

(b)

Figure 1. Location (a) and TripleSat image (b) of the study site near Novosady village, Minsk region. 
growing trees the forest pathologist found that most of them are also infected. There were also areas with spruces in the gray-attack stage and fallen dry spruce.

\subsection{Remote Sensing Data}

\subsubsection{Aerial Data}

\section{1) Equipment}

Small-sized photo-spectral equipment - spectrometers FSR-02 and SSP-600 [27] [28], operating in combination with survey cameras was applied in the research. The equipment was developed and designed at the A.N. Sevchenko Research Institute. Both spectrometers have a concave diffraction grating as a dispersing element and uncooled linear CCD detector Toshiba 1304DG. The FSR-02 spectrometer was installed on board the Diamond DA40NG aircraft (Figure 2), and the SSP-600 spectrometer was used for ground surveying of the diffuse plate. The technical characteristics of the spectrometers are presented in Table 1.

An interference filter was installed in front of the detector in FSR-02 spectrometer to protect the detector from undesired radiation of the second order of diffraction. Since there is no such filter in the construction of SSP-600 spectrometer,

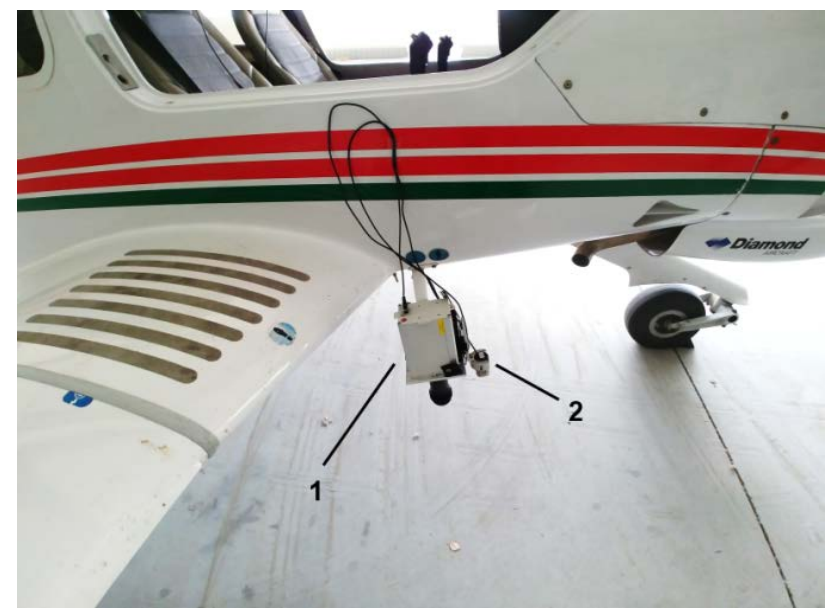

Figure 2. The FSR-02 spectrometer coupled with a survey camera installed on board the Diamond DA40NG aircraft: 1-FSR-02 spectrometer; 2-survey camera.

Table 1. Technical characteristics of FSR-02 and SSP-600 spectrometers.

\begin{tabular}{ccc}
\hline & \multicolumn{2}{c}{ Spectrometer } \\
\cline { 2 - 3 } Characteristic & SSP-600 & FSR-02 \\
\cline { 2 - 3 } Spectral range, $\mathrm{nm}$ & $400-900$ & $400-900$ \\
Spectral resolution (450 nm), nm & 4.5 & $1.5 \times 0.5$ \\
Angular field of view, & & 12 \\
ADC capacity, bit & $3 \times 0.5$ & 3648 \\
Number of pixels & 12 & +
\end{tabular}


the correction was performed by the calculation method according to the method [29] at the stage of preliminary data processing. Laptops with internally developed software were used for the purposes of control and data storage.

Defender WebCam G-Lens 2577 HD720p RGB survey camera was used for image registration during the airborne experiment. The survey camera has a resolution $640 \times 480$ pixels and a frame rate of $28 \mathrm{fps}$. Images of survey camera are linked to FSR-02 spectrometer's field of view. The field of view was measured in the laboratory with FSR-02 focused on infinity and was added by software on each image in the process of image recording. An automatic adjustment of photosensitivity, exposure and white balance was made during the photographing process since it is not possible to adjust the photography settings in the process of flight experiment. The FSR-02 spectrometer was also equipped with a Garmin GLO 2 portable GPS/GLONASS navigation module (10 $\mathrm{Hz}$ update rate) that provided spatial referencing of spectral data in the airborne experiment.

\section{2) Experiment}

On July 10, 2020 a field experiment was carried out for measuring spectral reflectance of an underlying surface of the test forest area under study. Continuous recording of the reflected radiation spectra was implemented from Diamond DA40NG aircraft using FSR-02 spectrometer, together with the corresponding imagery of Defender G-lens2577 survey camera. Airborne survey was performed at two heights above the underlying surface - $130 \mathrm{~m}$ and $450 \mathrm{~m}$.

Continuous quasi-synchronous ground recording of the diffuse plate spectra using SSP-600 spectrometer was being carried out along with airborne measurements to ensure the possibility of calculating spectral reflectance of the objects of underlying surface at each moment of time. It is assumed that the illumination of the horizontal surface at the ground level and at the level of tree crowns is the same. The brightness spectra of the diffuse plate and the airborne reflection spectra were recorded with a time resolution of $500 \mathrm{~ms}$. Figure 3 shows

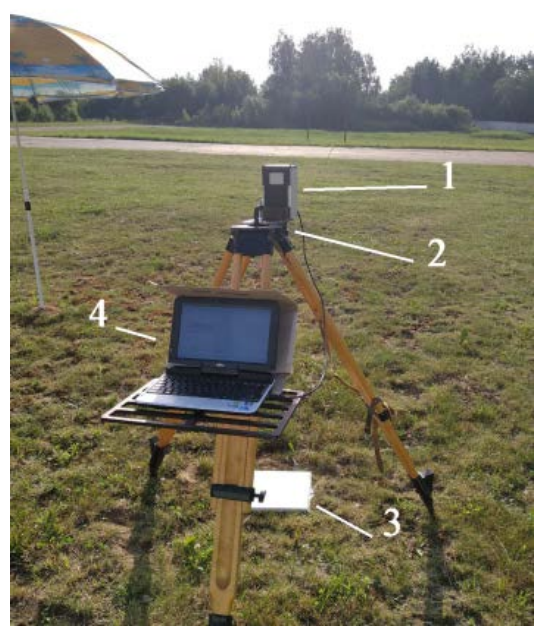

Figure 3. Device for diffuse plate spectra registration: 1-SSP-600 spectrometer; 2-a tripod with a bracket; 3 -diffuse plate; 4-laptop computer. 
the external view of field device for diffuse plate spectra registration; the device was located about $120 \mathrm{~m}$ away from the edge of the test forest area.

The duration of the airborne experiment was 1 hour 53 minutes, the average flight speed was $155 \mathrm{~km} / \mathrm{h}$. During the flight, reflection spectra and the corresponding images of the investigated forest area were recorded. Spectra in both airborne and field measurements were obtained in auto exposure mode, providing the best signal-to-noise ratio under conditions of varying signal intensity. The auto exposure algorithm consists in proportional correction of the exposure time of each subsequent recorded spectrum based on the intensity of the previously recorded spectrum, its exposure time and the optimal signal level. The optimal signal level was considered to be $70 \%$ of the saturation signal level of detector of spectrometer.

\subsubsection{Satellite Data}

The TripleSat Constellation [30] provides daily ultra-high resolution data with large area coverage. To study the possibility of determining drying conifers from satellite data, we used a commercial image of a TripleSat sensor with a spatial resolution of $3.2 \mathrm{~m}$ in 4 spectral channels Blue (0.44 - 0.51), Green (0.51 - 0.59), Red (0.60 - 0.67), Near Infrared (0.76 - 0.91) and $0.8 \mathrm{~m}$ in panchromatic, obtained on June 25, 2020. For the satellite image, the radiometric correction was performed using the ENVI 5.3 software. Atmospheric correction of the TripleSat image was not performed in order to eliminate the influence of inaccuracies associated with it on the classification results.

Pan sharpening procedure was performed for the multispectral TripleSat image using the nearest neighbor diffusion pan sharpening algorithm in the ENVI software, based on the corresponding high-resolution panchromatic image to increase the spatial resolution of the image. The pan-sharpened image with a spatial resolution of $0.8 \mathrm{~m}$ was used further in the study.

\section{Methods}

Identification of the drying out of conifers is based on a two-stage procedure using airborne and satellite data: control points with inherent stage of drying are identified based on results of airborne data classification, on the basis of which a supervised classification of the space image is carried out. At the first stage, reference points (objects) with known stages of drying and coordinates are determined from the reflection spectra and images of underlying surface obtained during the aviation experiment. At the second stage, the satellite image is classified with training sets using the reference points, the input data are a set of vegetation indices derived for each image pixel. The classification is carried out by the method of LDA for assignment to such types of underlying surface as "drying out spruce", "dead-wood", "vigorous spruce”, "undergrowth", "soil”. On the basis of classification results, types of underlying surface have been mapped. The sequence of procedures used in this work is presented in the form of a flowchart in Figure 4. 


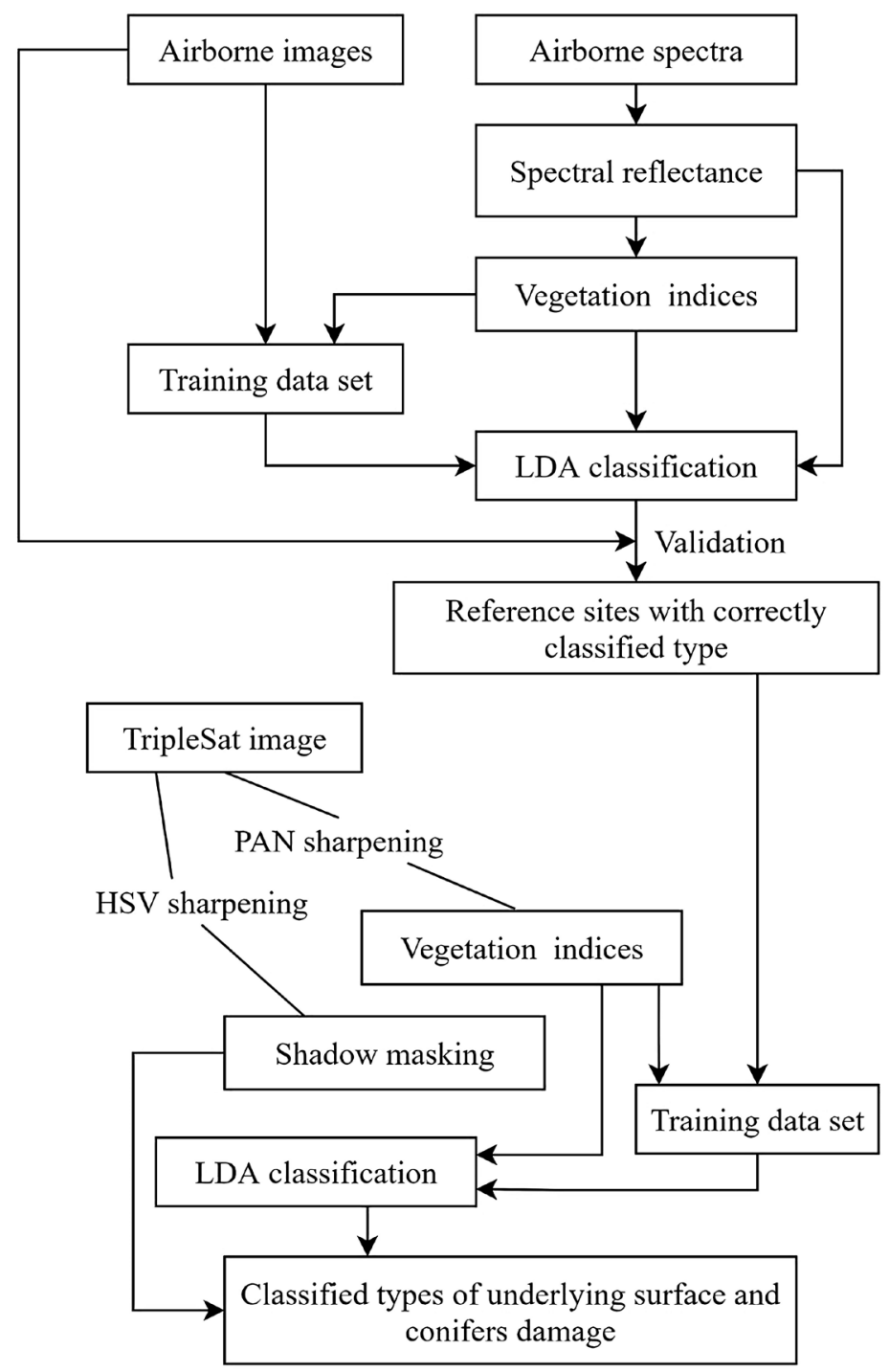

Figure 4. Methodological flowchart for determining the drying out of conifers.

\subsection{Techniques for Handling Aviation Data}

\subsubsection{Pre-Processing of Spectral Data. Spectral Reflectance Calculation}

The preliminary processing of the reflection spectra consisted in spectrometer wavelength calibration using mercury lines and subsequent radiometric calibration. Radiometric correction was carried out in order to convert the spectral data in relative units $(\mathrm{DN})$ to the absolute units of spectral radiance. To calculate the spectral reflectance using formula (1) given below, data from both SSP-600 and FSR-02 spectrometers should be represented in the same units of spectral radiance. Both wavelength and radiometric calibration of spectrometers were performed in laboratory using a photometric complex [31].

The second-order diffraction signal was corrected for the data of the SSP-600 spectrometer. The algorithm of the second-order diffraction correction is based on the decomposition of the spectrum by spectrometer's slit functions. The second-order diffraction signal proportional to the amplitude of the slit function 
in the first diffraction order that caused it [29] is then subtracted from initial spectrum. To determine spectrometer's slit functions depending on the wavelengths, the spectra of a mercury lamp and laser diodes with known narrow emission lines $(406.9,435.8,446.37,516.37 \mathrm{~nm})$ were previously registered in the laboratory. Figure 5 shows the result of applying the algorithm of the second-order diffraction correction for the brightness spectrum of a section of celestial sphere recorded by the SSP-600 spectrometer.

The uncorrected spectrum has a clearly distinguishable signal in the 700 $1000 \mathrm{~nm}$ range, representing an effect of aliasing the second-order diffraction spectrum (generated from the first-order diffraction spectrum with a maximum at $480 \mathrm{~nm}$ ) into true spectrum. As shown in Figure 5, the spectrum with correction matches the one registered through the KS10 filter in the wavelength region above $650 \mathrm{~nm}$, confirming the validity of the algorithm of correction. The spectrum registered through the KS10 filter is a true reflection spectrum of an object in the 650 - $1000 \mathrm{~nm}$ range that is not affected by higher diffraction orders due to suppression of reflected radiation with a wavelengths less than $600 \mathrm{~nm}$.

The reflectivity of the object under study [32] was calculated by the formula:

$$
\operatorname{SR}\left(\lambda_{i}\right)=\frac{B_{\mathrm{FSR}}\left(\lambda_{i}\right)}{B_{\mathrm{SSP} \_ \text {DP }}\left(\lambda_{i}\right)}
$$

where $\lambda_{i}$ is the wavelength; $B_{\mathrm{FSR}}\left(\lambda_{i}\right)$-spectral radiance of the object under study, measured by the FSR-02 spectrometer; $B_{\text {SSP DP }}\left(\lambda_{i}\right)$-spectral radiance of diffuse plate measured by SSP-600. Spectral radiance $B$ was calculated by the formula:

$$
B(\lambda)=\left\{\begin{array}{cl}
k\left(\frac{I(\lambda)}{\exp }\right)^{4}+b\left(\frac{I(\lambda)}{\exp }\right)^{3}+c\left(\frac{I(\lambda)}{\exp }\right)^{2}+d\left(\frac{I(\lambda)}{\exp }\right)+e, & \text { if } \frac{I(\lambda)}{\exp } \leq \text { max_sign }(\lambda) \\
k_{1} \frac{I(\lambda)}{\exp }+k_{2}, & \text { if } \frac{I(\lambda)}{\exp }>\text { max_sign }(\lambda)
\end{array}\right.
$$

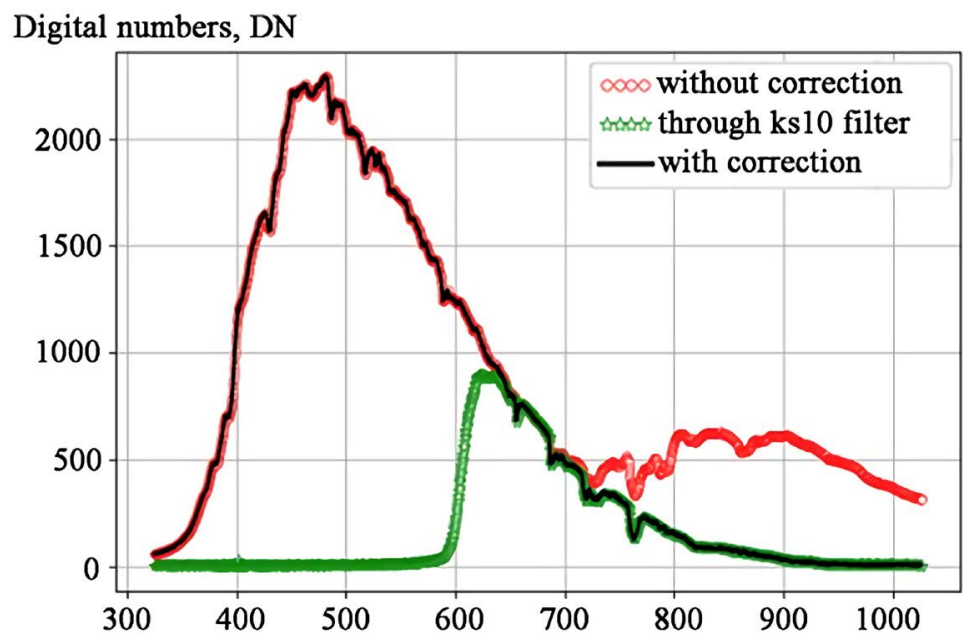

Figure 5. Result of second-order diffraction correction for SSP-600 spectrum of the section of celestial sphere. 
where $I(\lambda)$ is the intensity of the signal at the wavelength $\lambda$ in DN units; $\exp$-exposure of the spectrum in $\mathrm{ms}$;

$k_{1}, k_{2}$ and $k, b, c, d, e$-the coefficients of the polynomials of the first and fourth degrees, respectively, determined during the calibration on the photometric sphere in the laboratory;

$\max \_\operatorname{sign}(\lambda)$ - the maximum signal level, normalized to the exposure time, recorded by the spectrometer at a wavelength $\lambda$ while performing an absolute radiometric calibration.

The coefficients $k, b, c, d, e, k_{1}, k_{2}$ and $\max \_\operatorname{sign}(\lambda)$ values were determined for the SSP-600 spectrometer from the spectra of the photometric sphere corrected for the removal of second orders during the calibration process. Formula (2) actually takes into account the nonlinearity of the detector response depending on the brightness of the photometric sphere by means of a 4th degree polynomial. The values obtained in field measurements were also subjected to radiometric calibration for the SSP-600 spectrometer.

Figure 6(a) shows the examples of the diffuse plate spectra of the SSP-600 at different moments of registration after the second-order diffraction signal correction. An example of the reflection spectrum of an object recorded using the

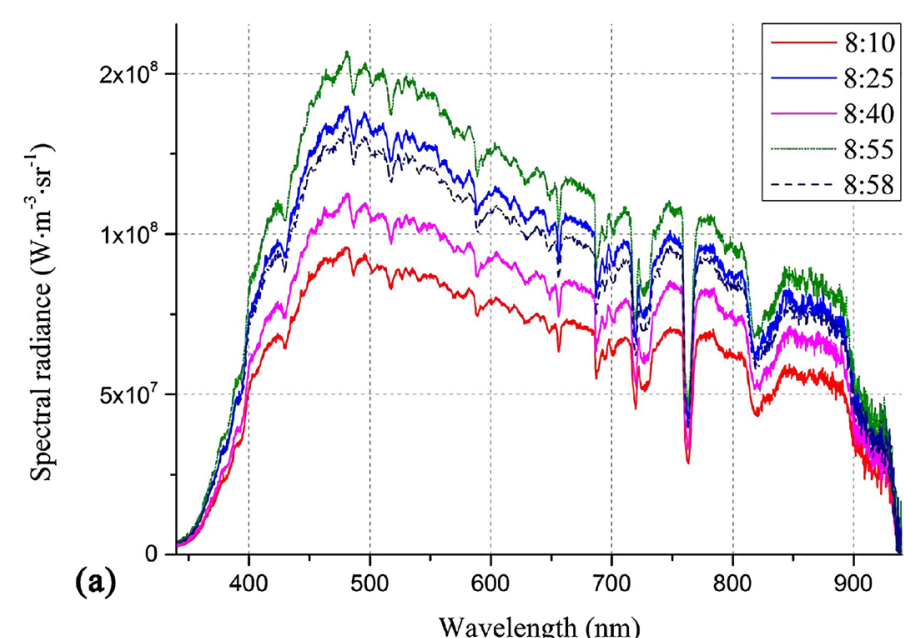

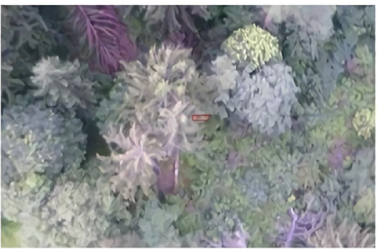

(b)

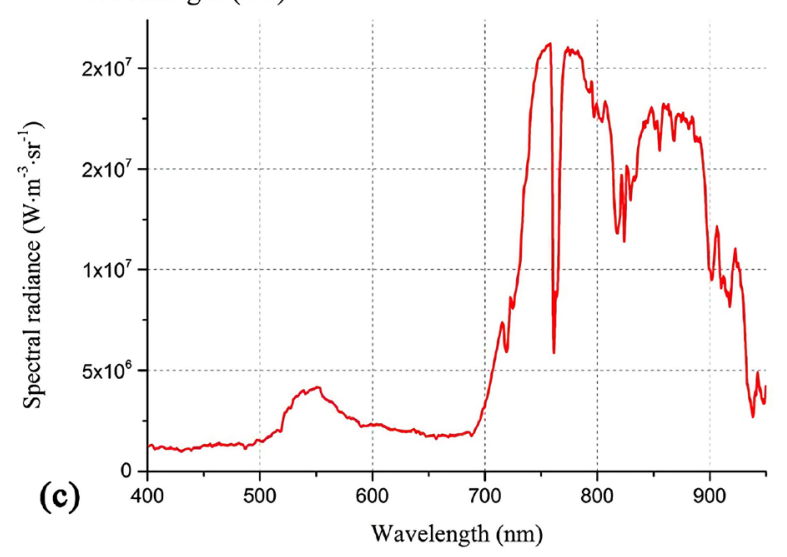

Figure 6. Diffuse plate spectra of SSP-600 at different moments of registration (a), image of a survey camera with an FSR-02 field of view (b), spectrum of FSR-02 (c). 
FSR-02 is shown in Figure 6(c), and the corresponding image of the survey camera with the FSR-02 field of view is shown in Figure 6(b).

\subsubsection{Evaluation of Vegetation Indices}

58 different vegetation indices were evaluated for each spectral reflectance dependency obtained over the investigated forest area. The following indices were chosen [33] [34]: ARVI, MCARI, MCARI2, MRENDVI, MRESR, MTVI, MTVI2, RENDVI, TCARI, TVI, VREI1, PRI531/570, PSRI, REP, NDVI, Datt1, DVI, SR750/700, GNDVI, mSR, SIPI1, LIC2, SR440/740, SR750/550, Ctr2, MD734/747/715/720, ARI2, CRI1, GNDVIhyper, D800/550, NPCI, Vog2, Ctr3, Ctr4, PSNDa1, PSNDb1, PSSRa1, PSSRb1, PSSRc2, RARS, SR675/700, SR800/680, as well as 16 indices developed according to previously conducted laboratory studies [35]. The indices were chosen as the ones sensitive to the content of chlorophyll and other pigments in vegetation.

\subsubsection{Training Sample Formation for Aerial Data}

The training sample was formed from sets of vegetation indices for the surface types "vigorous vegetation", "drying out spruce", "dead-wood", "undergrowth" and "soil" for the purposes of supervised classification. The above types of underlying surface were chosen as the most represented among the forest area under study and suitable for visual identification using available aerial images. Characteristic objects were selected for inclusion in the training sample by visual analysis of the corresponding aerial images; the particular features of the process are described below.

When shooting from a moving vehicle, each recorded reflection spectrum is an averaged spectrum of a certain area of the territory. Thus, for an average exposure time of $100 \mathrm{~ms}$ at a speed of about $50 \mathrm{~m} / \mathrm{s}$, the aircraft covered a distance of about $5 \mathrm{~m}$, which approximately corresponded to the crown width of a mature tree. In this regard, two images closest to the times of the beginning and the end of spectrum registration were considered in the process of training sample formation. A decision on including the registered object in one of the classes of the training sample was made by visual image interpretation, namely: during the exposure time the spectrum of the object belonging largely to a single class should be recorded.

It was found out that it is preferable to use aerial data obtained at $130 \mathrm{~m}$ rather than at $450 \mathrm{~m}$ in the process of training sample formation, since the objects and details on the associated image are more distinguishable at low altitudes. The resolution of the airborne image is a determining factor for high-quality visual separation of objects of the class "drying out spruce" from objects of the class "vigorous vegetation", the separation is done by detecting the thinned state of the crown and occasionally the yellowing of the crown.

It should be noted that the values of spectral reflectance of objects of underlying surface should range from 0 to 1 . Nevertheless, there were a few spectral reflectance curves obtained during aerial experiment in which maximum values 
were $>1$ - as a rule, with a wavelength of more than $850 \mathrm{~nm}$. This is due to the influence of variable cloudiness, since the spectral measurements of the object and the diffuse plate were carried out at different locations and, despite the quasi-synchronous measurements, this could lead to differences in lighting conditions. Such spectral reflectance dependencies were excluded from further processing.

The classes of the training set were presented as the sets of vegetation indices and served then as training data for LDA supervised classification.

\subsubsection{LDA Classification}

The classification of each spectrum registered during aerial experiment was carried out using LDA method [36] based on the formed training sample containing sets of vegetation indices for different classes. As a part of its operation, the algorithm determined linear combinations of features (vegetation indices) that best separate the formed classes of objects. The resulting combinations were then used as a linear classifier for all aerial spectral data obtained.

The classification results were validated by comparison with the data of visual analysis of airborne images. Successful validation results (i.e. coordinates and surface class) were used as reference data for the satellite image classification.

\subsection{Techniques for Handling Satellite Data}

\subsubsection{Satellite Data and Preprocessing}

The TripleSat Constellation [30] provides daily ultra-high resolution data with large area coverage. To study the possibility of determining drying conifers from satellite data, we used a commercial image of a TripleSat sensor with a spatial resolution of $3.2 \mathrm{~m}$ in 4 spectral channels Blue (0.44 - 0.51), Green (0.51 - 0.59), Red $(0.60-0.67)$, Near Infrared $(0.76-0.91)$ and $0.8 \mathrm{~m}$ in panchromatic, obtained on June 25, 2020. For the satellite image, the radiometric correction was performed using the ENVI 5.3 software. Atmospheric correction of the TripleSat image was not performed in order to eliminate the influence of inaccuracies associated with it on the classification results.

Pan sharpening procedure was performed for the multispectral TripleSat image using the nearest neighbor diffusion pan sharpening algorithm in the ENVI software, based on the corresponding high-resolution panchromatic image to increase the spatial resolution of the image. The pan-sharpened image with a spatial resolution of $0.8 \mathrm{~m}$ was used further in the study.

\subsubsection{Shadow Masking}

The presence of shadow pixels among the objects of the training sample of the satellite image can subsequently cause inaccuracies in the classification results [37]. Shadow pixels should be attributed to a separate class and excluded from further processing. For this purpose, the HSV (Hue, Saturation, Value) Pan Sharpening procedure was applied to the Red, Green and Blue TripleSat bands and thus the image was transformed into the space of value (Hue, Saturation, 
Value). It was found out that shadows correspond to Value equal to less than $10 \%$ of the maximum level of the dynamic range of image band values in (Hue, Saturation, Value) space, i.e. Value $<25$ corresponds to shadows at the maximum value 255. A shadow mask was compiled by evaluating the value of each image pixel for matching the conditions of shadow pixels.

\subsubsection{Calculation of Vegetation Indices}

A set of 11 vegetation indices was calculated for each pixel of the pan-sharpened TripleSat image, including: MCARI2, MTVI, MTVI2, SIPI1, LIC2, GNDVIhyper, D800/550, NPCI, PSNDa1, PSSRa1, PSSRc2. The indices were selected from those listed in paragraph 3.1.2, taking into account the possibility of calculating them for TripleSat bands.

\subsubsection{Training Sample Formation for Satellite Image}

The control points resulting from the airborne experiment were used to form the training sample for TripleSat image classification. Using geographic coordinates of the control points, as well as corresponding images of the survey camera, these points were selected in the TripleSat image, avoiding the shadow pixels according to the shadow mask. The LDA supervised classification algorithm was applied to each pixel of the TripleSat image, sets of vegetation indices were used as input data. The results of classification were mapped then.

\section{Results}

\subsection{Results of the Airborne Experiment}

Figure 7 shows spectral reflectance of spruce with different degrees of drying out, as well as undergrowth (shrubs and grass cover), according to the results of airborne measurements. Spectral reflectance of drying spruce are generally characterized by a decrease in reflection values in the near-IR region $(760-900 \mathrm{~nm})$

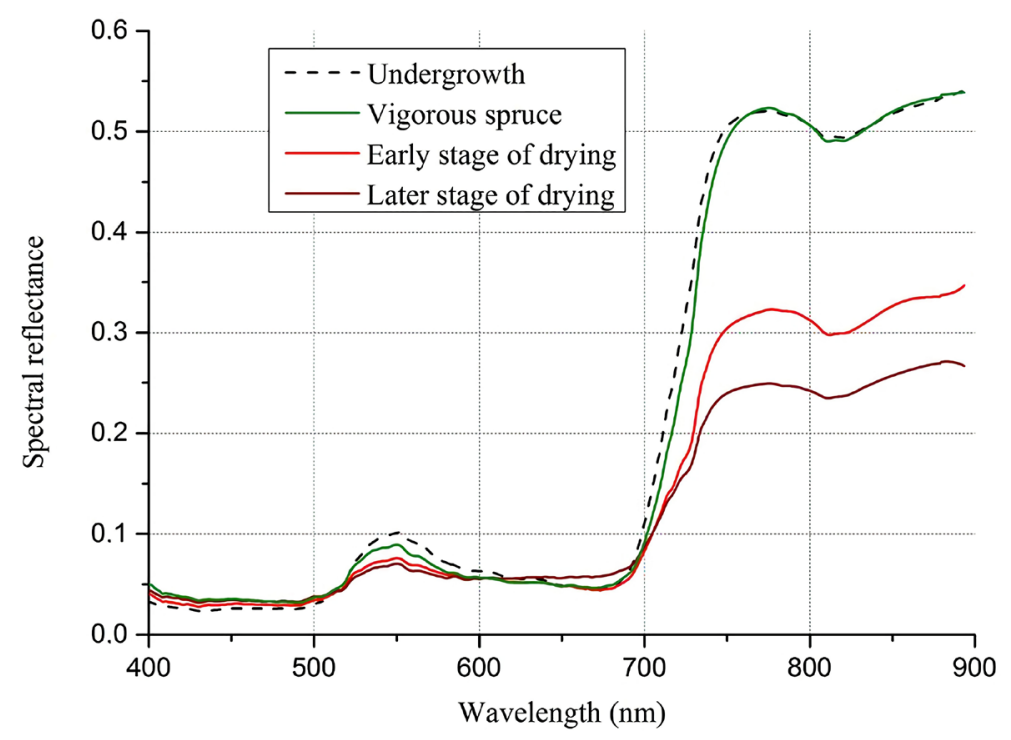

Figure 7. Spectral reflectance of various objects by airborne measurements. 
and in the range of $520-570 \mathrm{~nm}$ and also an increase in reflection in red region $(600-680 \mathrm{~nm})$. The results are consistent with the existing in the literature features of reflectivity of green-attack stage conifers [15]. The spectral reflectance dependences of vigorous spruce and undergrowth are similar to each other, except the undergrowth has a higher reflectance in the range of $520-570 \mathrm{~nm}$ than vigorous spruce.

The number of reflectance spectra constituting the training set was $20 \%$ of the total number of spectral reflectance curves recorded over the studied area which in turn was 249 pcs. The remaining $80 \%$ of the spectra were left as testing sample.

For each of the spectral reflectance curves of the training sample, 58 vegetation indices were calculated, according to paragraph 3.1.2. When presenting the training sample data in a two-dimensional space of selected pairs of vegetation indices, it was noticed that objects of the same classes tend to group (clustering). More than 1200 different combinations of vegetation indices were analyzed to determine the combination of indices for which objects of the same classes correlate the best. In particular, in the space of vegetation indices PRI531/570 and LIC2, objects of the training set class "drying out spruce" constituted a separate cluster (Figure 8), along with clusters "dead-wood", "vigorous vegetation", etc., and moreover, the cluster "drying out spruce" is located at the junction of the clusters "vigorous vegetation" and "dead-wood". In Figure 8, each point of a certain color corresponds to the object of a certain class of the training sample. Vegetation indices PRI531/570 and LIC2 are known as chlorophyll-sensitive indices for detecting plant stress and are calculated by the formulas:

$$
\text { PRI 531 } / 570=\frac{R_{531}-R_{570}}{R_{531}+R_{570}}, \mathrm{LIC} 2=\frac{R_{440}}{R_{690}}
$$

where $R_{\lambda}$ is the value of spectral reflectance at a wavelength $\lambda$.

While spectral reflectance of the undergrowth and vigorous vegetation are practically similar to each other in Figure 7, Figure 8 clearly shows the differences

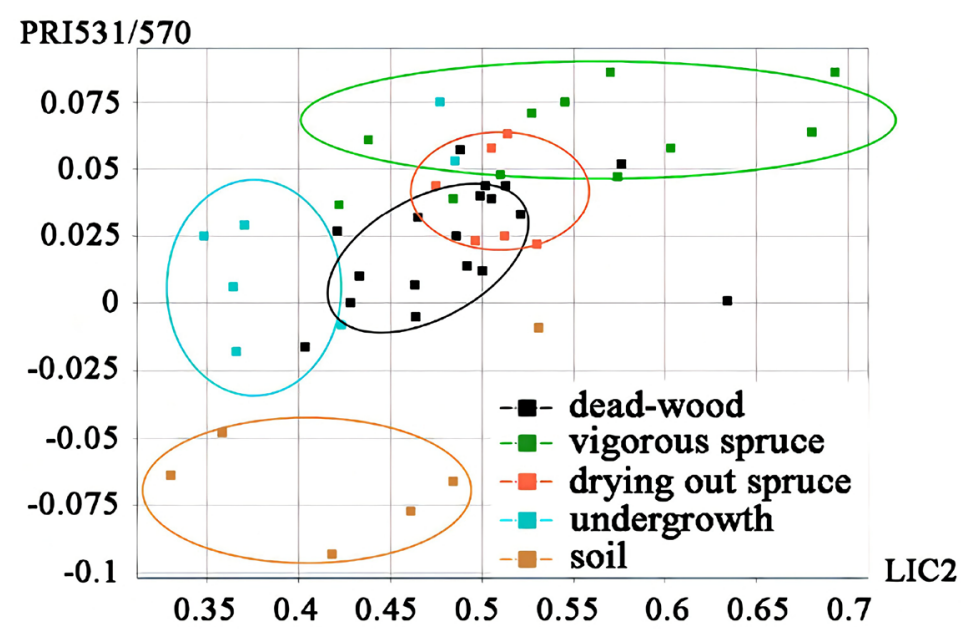

Figure 8. Scatter plot for PRI531/570 (LIC2) indices of the training sample. 
between the clusters "vigorous vegetation" and "undergrowth". The vegetation indices are therefore a more significant characteristic of the classes than original reflectance dependences. The representation of the training sample data in the form of points of the two-dimensional space of the vegetation indices can serve as a simple classifier.

The LDA classification algorithm was applied to the formed set of 58 vegetation indices calculated for the objects of the training sample. Thus, a classifier was formed, the application of which to the entire array of the airborne data makes it possible to obtain information about the attribution of a particular spectrum to a certain class. The result of the classification plotted on the ESRI World Imagery map [38] is shown in Figure 9(b), where points of various colors correspond to various classes of the underlying surface. The result of spatial interpolation of the obtained values to the study area is shown in Figure 9(c). The class values were interpolated by the method of nearest neighbors on a uniform coordinate grid of $1000 \times 1000$, followed by the application of a Gaussian filter for smoothing.

Analyzing the data presented in Figure 9, it can be concluded that there are

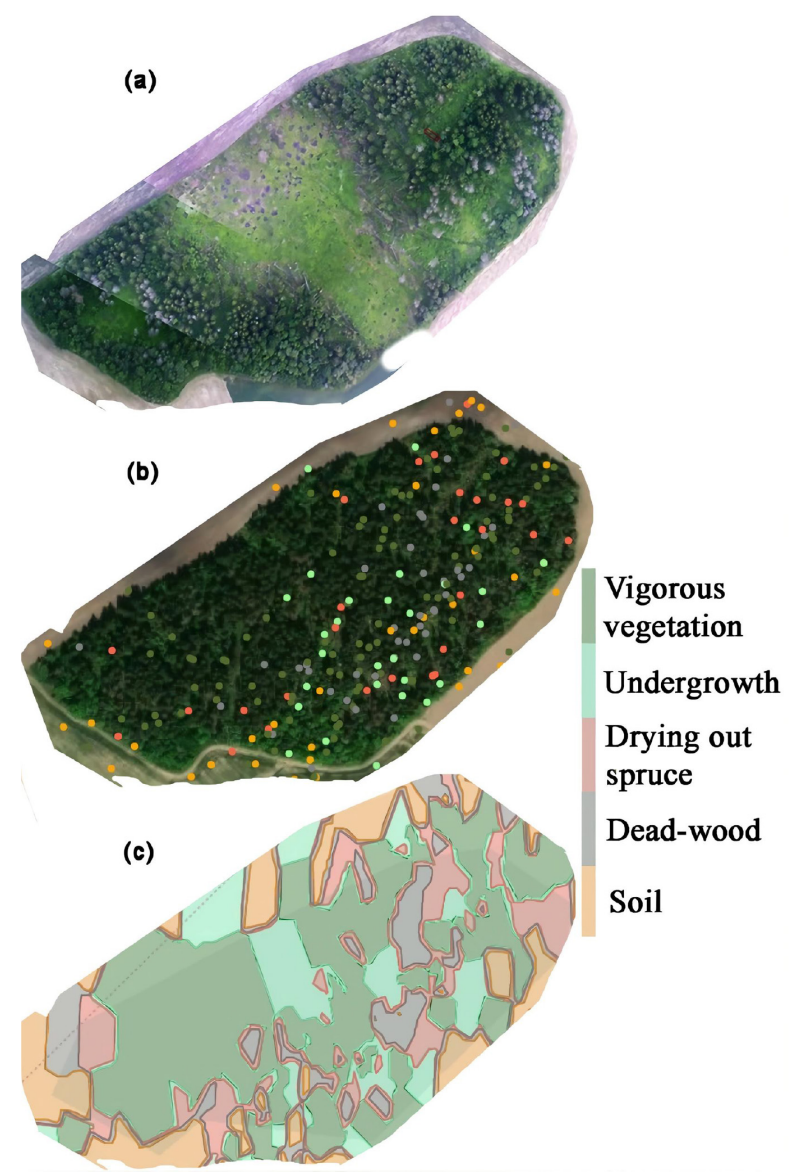

Figure 9. The result of the classification of airborne reflection spectra of the underlying surface by the LDA supervised classification: image of the survey camera (a), values at the points of survey (b), spatial interpolation of class values (c). 
correspondences of the classified objects to those in the airborne image of the survey camera: vast areas of dead wood among the clearings and dry conifers are identified mainly as "dead-wood", and the area of fields around the forest where only rare vegetation presented was classified as "soil". It is noticeable that the class "undergrowth" is mostly present among the clearing in the middle of the forest area, and the trees practically did not fall into this class, which also indicates the ability of the obtained classifier to distinguish the undergrowth from other plant objects.

To check the quality of the classification, the photographic images corresponding to 249 flight spectra were analyzed by the method of visual recognition of the types of underlying surface. The result of the visual estimation of the types of underlying surface was then compared with the result of the LDA classification, the confusion matrix is presented in Table 2.

It became possible to divide 211 airborne images (out of 249) into classes designated as "Reference values" in Table 2. The best classification results are observed for the classes "soil" (78\%) and "vigorous vegetation" (74\%). The classification accuracy for the "drying out spruce" class was $27 \%$. In case of misclassification, the "drying out spruce" class, like the "undergrowth" class, was most often wrongly assigned to the "vigorous vegetation" class.

\subsection{Results of TripleSat Image Classification}

The aerial experiment spectra that have successfully passed the classification check, the number of which are presented as diagonal elements in Table 2, can be interpreted as reference points with known types of underlying surface and corresponding geographic coordinates. The reference points defined in this way are used as a ground truth information for the formation of a training sample and the subsequent classification of the satellite image. The result of the supervised classification of the TripleSat image of the study area with shadow mask applied is shown in Figure 10(b).

It can be seen from Figure 10 that the "vigorous vegetation" class is present not only in the considered forest area, but also in the area corresponding to the

Table 2. LDA classification confusion matrix.

\begin{tabular}{ccccccc}
\hline \multirow{2}{*}{$\begin{array}{c}\text { Reference } \\
\text { values }\end{array}$} & $\begin{array}{c}\text { Drying out } \\
\text { spruce }\end{array}$ & $\begin{array}{c}\text { Vigorous } \\
\text { vegetation }\end{array}$ & Soil & Undergrowth & Dead-wood & Total \\
\cline { 2 - 6 } & $8(27 \%)$ & 18 & 1 & 1 & 2 & 30 \\
\hline $\begin{array}{c}\text { Drying out spruce } \\
\text { Vigorous vegetation }\end{array}$ & 2 & $20(74 \%)$ & 3 & 0 & 2 & 27 \\
Soil & 0 & 4 & $49(78 \%)$ & 9 & 1 & 63 \\
Undergrowth & 6 & 24 & 2 & $20(31 \%)$ & 12 & 64 \\
Dead-wood & 1 & 9 & 0 & 4 & $34 \%$ & 27 \\
\hline
\end{tabular}




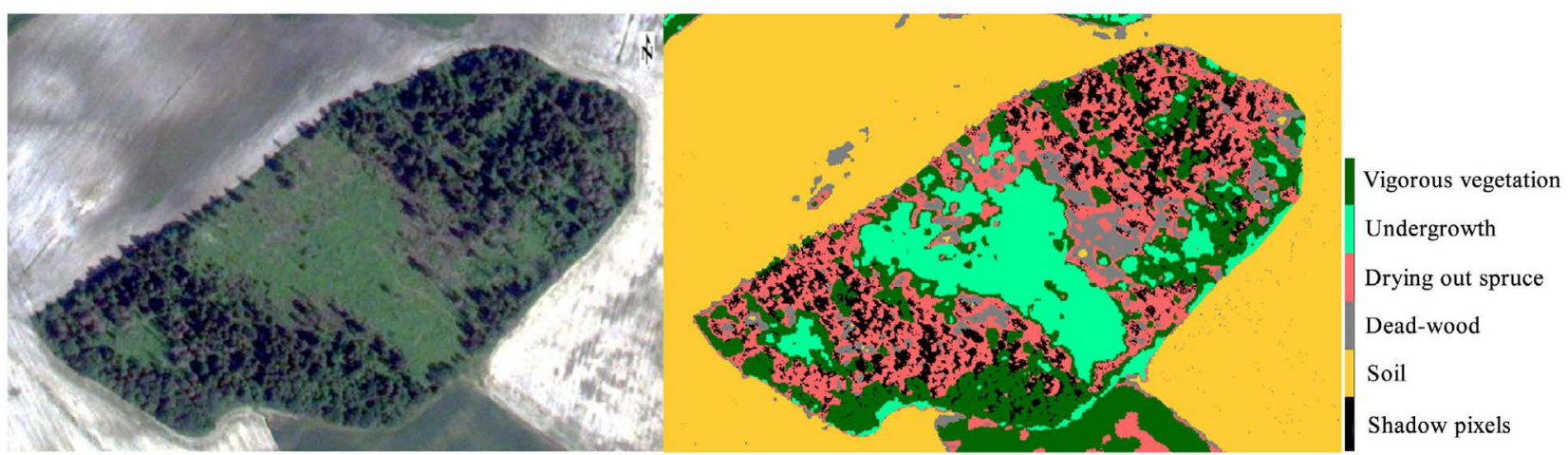

(a)

(b)

Figure 10. The result of the classification of TripleSat image by the LDA supervised classification: pan sharpened TripleSat image (a), class values at image points (b).

corn field, at the bottom of the image, which indicates the fact that the algorithm does not distinguish between vigorous tree species and meadow vegetation. There are rare pixels corresponding to the soil cover and incorrectly classified as "dead-wood" or "drying out spruce".

Such types of underlying surface as "soil", "undergrowth" and "dead-wood" were determined mainly correctly using LDA classification of the satellite image, as can be seen by comparing the classification results presented in Figure 10 with the image of the survey camera in Figure 9(a).

\section{Discussion}

\subsection{Accuracy of Classification}

The accuracy of the classification of the stages of spruce drying out was estimated quantitatively for an airborne experiment. The classification accuracy for different stages of spruce drying was as follows: "drying out spruce" $27 \%$, "vigorous vegetation" $74 \%$, "dead-wood" $48 \%$. Insufficient accuracy of classification of the stages of spruce drying out is associated with inaccuracies in georeferencing, small sizes of the objects under study and also blur due to aircraft movement. The classification accuracy was $78 \%$ for the "soil" class being presented by extended objects that largely excludes georeferencing errors. In case of misclassification, the class "drying out spruce" was most often wrongly assigned to the class "vigorous vegetation" due to the similarity of the reflection spectra of objects of these classes and also blur. It should be noted that the visual method for verifying the classification results can significantly distort the classification accuracy evaluation due to impossibility of accurately delineation the stage of spruce drying out with aerial images.

The classification accuracy for the satellite image was assessed qualitatively by visual comparing the classification results with aerial images of the same territory. For this purpose, the southern section of the investigated forest area was selected (Figure 11). The airborne frames of the RGB camera of this area were combined into a mosaic, shown in Figure 11(a), which shows spruce trees, deciduous 


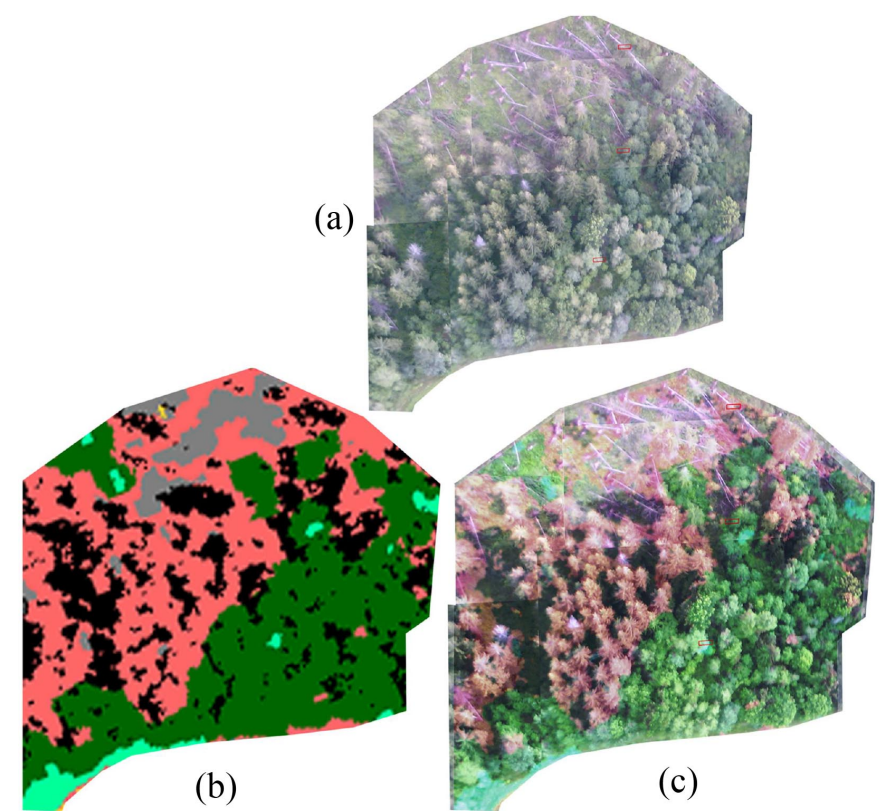

Figure 11. Mosaic of airborne frames (a); result of classification of the TripleSat image (b); classification results layer overlaid on the mosaic of territory (with some transparency) (c).

trees, fallen spruce trees, and areas of undergrowth. Result of LDA classification is shown in Figure 11(b), the classification results layer overlaid on the mosaic of the territory (with some transparency) is shown in Figure 11(c). From Figure 11(c), one can see a clear distinction between deciduous trees, which are included in the "vigorous vegetation" class, and spruces, which are included in the "drying out spruce" class. The area of fallen trees corresponds to the classes "dead-wood" and "drying out spruce". Shaded areas correspond to areas between trees where shading naturally occurs. Due to the lack of additional a priori data on the state of health of trees and the impossibility of identifying vigorous spruce trees from the mosaic image, there is no currently way to verify the correctness of classification of the types "drying out spruce" and "vigorous vegetation".

Thus, having images of the aerial camera for reference, it is possible to draw only a qualitative conclusion about the generally satisfactory result of the TripleSat image classification of the stages of spruce drying. To quantify the accuracy of the satellite image classification, higher spatial resolution images and up-to-date information from the databases of forest pathological services on the health status of individual trees are required.

\subsection{Aerial Data}

It is shown in the study that the data of the spectrometers used in the airborne experiment are suitable for distinguishing the stages of drying out of spruce trees. The advantages of spectrometers are a high spectral resolution, a wide range of registered wavelengths, and, as a consequence, it is possible to obtain 
complete spectral information in the range of $400-900 \mathrm{~nm}$ about the objects of study. As known from the literature, visible and near-IR ranges of detected radiation play a crucial role in identifying the stages of coniferous desiccation. Combination of the reflectance spectra of objects and the corresponding photo-images provides more opportunities in terms of recognizing the stages of drying out than using them separately. At the same time, the calculation of vegetation indices allows for an even better delineation of the stages of drying out of spruce.

Registration of spectra of the underlying surface from an airplane cause significant inaccuracies in the subsequent interpretation of airborne data, since the blur arising from the motion of the airplane worsens the spatial resolution of the spectral data. In this regard a possible solution in future research could be the use of an unmanned aerial vehicle with a lower movement rate.

\subsection{TripleSat Data}

The results of the classification (Figure 10(b)) show that the majority (about $90 \%$ ) of the forest area under study is in the drying stage, with slight inclusions of healthy trees. This fact is well consistent with the forest pathologist expertise about that there are almost no healthy trees in the study area and it is subjected to regular sanitary cuttings due to bark beetle infestation.

The study of a forest of a relatively small area carried out in this work entails the determination of the stages of drying out of individual trees, which can only be carried out using satellite data of ultra-high spatial resolution. At the same time, from the results of the airborne experiment presented in Table 2, it can be seen that there is a correct separation of the "soil" and "dead-wood" classes from each other. On the satellite image there are soil areas classified as "dead-wood" or "drying out spruce" (Figure 10(b)). Hence, it can be concluded that the incorrect assignment of the TripleSat image pixels corresponding to the soil to the "dead-wood" or "drying out spruce" classes is obviously due to the lack of spectral resolution of the TripleSat sensor.

Atmospheric correction of the TripleSat image was not applied in the study in order to avoid inaccuracies associated with it. Radiometric correction was applied. Nevertheless, both radiometric and atmospheric correction should be applied for developing a general methodology for the classification of drying stages from Triplesat images. Only pixels corresponding to the sunlit parts of trees were used in classification, and shadow pixels were excluded by applying a shadow mask, which made it possible to reduce the variability of classes of training sample.

The accuracy of classification of dying spruce trees using TripleSat data was found to be satisfactory.

\section{Conclusion}

The study presents the results of determining the three stages of the state of 
spruce (vigorous, drying out spruce and dead wood) also as two additional types of underlying surface (soil, undergrowth) based on the classification of the TripleSat image by vegetation indices. The reference data for the classification were the results of the airborne experiment. Using vegetation indices calculated from the data of airborne spectrometers in a wide spectral range of $400-900 \mathrm{~nm}$, it is possible to identify three stages of drying out spruce. The accuracy of determining the stages of drying out in an airborne experiment is: "vigorous vegetation" $-74 \%$, "drying out needles"- $27 \%$, "dead wood" $-48 \%$. The accuracy of the TripleSat image classification by the LDA method was assessed as satisfactory by comparing the classification maps with the aerial images. To determine more accurately the drying out of spruce and access the classification accuracy, future studies should use an unmanned aerial vehicle with a lower speed of movement, high quality photographic images of forest area, and detailed data from forest pathological services on the presence of forest diseases.

\section{Conflicts of Interest}

The authors declare no conflicts of interest regarding the publication of this paper.

\section{References}

[1] National Environmental Monitoring System (2019) Forest Monitoring Results for 2019. https://www.nsmos.by/content/176.html

[2] Sarnatskiy, V. (2009) Spruce Forests: Development, Raising of Productivity and Stability in Belarus. “Technology” Press, Minsk. (In Russian)

[3] Bond-Lamberty, B., Rocha, A., Calvin, K., Holmes, B., Wang, C. and Goulden, M. (2013) Disturbance Legacies and Climate Jointly Drive Tree Growth and Mortality in an Intensively Studied Boreal Forest. Global Change Biology, 20, 216-227. https://doi.org/10.1111/gcb.12404

[4] Langstrom, B., Lindelöw, Å., Schroeder, M., Björklund, N. and Öhrn, P. (2009) The Spruce Bark Beetle Outbreak in Sweden Following the January-Storms in 2005 and 2007. IUFRO Forest Insect and Disease Survey in Central Europe, Slovakia, 15-19 September 2008, 1-8.

[5] Harmon, M.E. and Pabst, R.J. (2019) The Long-Term Effects of Wind Disturbance on a Sitka Spruce-Western Hemlock Forest. Forests, 10, 119. https://doi.org/10.3390/f10020119

[6] Allen, C.D., Macalady, A.K., Chenchouni, H., et al. (2010) A Global Overview of Drought and Heat-Induced Tree Mortality Reveals Emerging Climate Change Risks for Forests. Forest Ecology and Management, 259, 660-684. https://doi.org/10.1016/j.foreco.2009.09.001

[7] Stephens, S.L., Collins, B.M., Fettig, C.J., Finney, M.A., Hoffman, C.M., Knapp, E.E., North, M.P., Safford, H. and Wayman, R.B. (2018) Drought, Tree Mortality, and Wildfire in Forests Adapted to Frequent Fire. BioScience, 68, 77-88. https://doi.org/10.1093/biosci/bix146

[8] Netherer, S., Panassiti, B., Pennerstorfer, J. and Matthews, B. (2019) Acute Drought Is an Important Driver of Bark Beetle Infestation in Austrian Norway Spruce Stands. Frontiers in Forests and Global Change, 2, Article 39. 
https://doi.org/10.3389/ffgc.2019.00039

[9] Boczoń, A., Kowalska, A., Ksepko, M. and Sokołowski, K. (2018) Climate Warming and Drought in the Bialowieza Forest from 1950-2015 and Their Impact on the Dieback of Norway Spruce Stands. Water, 10, 1502. https://doi.org/10.3390/w10111502

[10] CABI (2021) Invasive Species Compendium. https://www.cabi.org/isc

[11] General Characteristics of the Forest Pathological Situation in the Forest Fund of the Republic of Belarus (2019) Spruce Forest.

https://bellesozaschita.by/front/ru/index?id=151

[12] Synek, M., Janda, P., Mikoláš, M., et al. (2020) Contrasting Patterns of Natural Mortality in Primary Picea Forests of the Carpathian Mountains. Forest Ecology and Management, 457, 117734. https://doi.org/10.1016/j.foreco.2019.117734

[13] Grodzki, W. (2016) Mass Outbreaks of the Spruce Bark Beetle Ips typographus in the Context of the Controversies around the Białowieża Primeval Forest. Forest Research Papers, 77. https://doi.org/10.1515/frp-2016-0033

[14] Hlásny, T., Krokene, P., Liebhold, A., et al. (2019) Living with Bark Beetles: Impacts, Outlook and Management Options. From Science to Policy 8. European Forest Institute. https://doi.org/10.36333/fs08

[15] Wulder, M.A., White, J.C., Carroll, A.L. and Coops, N.C. (2009) Challenges for the Operational Detection of Mountain Pine Beetle Green Attack with Remote Sensing. The Forestry Chronicle, 85, 32-38. https://doi.org/10.5558/tfc85032-1

[16] Wulder, M.A., Dymond, C.C., White, J.C., Leckie, D.G. and Carroll, A.L. (2006) Surveying Mountain Pine Beetle Damage of Forests: A Review of Remote Sensing Opportunities. Forest Ecology and Management, 221, 27-41.

https://doi.org/10.1016/j.foreco.2005.09.021

[17] Meddens, A.J.H., Hicke, J.A., Vierling, L.A. and Hudak, A.T. (2013) Evaluating Methods to Detect Bark Beetle-Caused Tree Mortality Using Single-Date and Multi-Date Landsat Imagery. Remote Sensing of Environment, 132, 49-58. https://doi.org/10.1016/j.rse.2013.01.002

[18] White, J.C., Wulder, M.A., Brooks, D., Reich, R. and Wheate, R.D. (2005) Detection of Red Attack Stage Mountain Pine Beetle Infestation with High Spatial Resolution Satellite Imagery. Remote Sensing of Environment, 96, 340-351.

https://doi.org/10.1016/j.rse.2005.03.007

[19] Immitzer, M. and Atzberger, C. (2014) Early Detection of Bark Beetle Infestation in Norway Spruce (Picea abies, L.) Using WorldView-2 Data. PhotogrammetrieFernerkundung-Geoinformation, 5, 351-367. https://doi.org/10.1127/1432-8364/2014/0229

[20] Liang, L., Chen, Y., Hawbaker, T., Zhu, Z. and Gong, P. (2014) Mapping Mountain Pine Beetle Mortality through Growth Trend Analysis of Time-Series Landsat Data. Remote Sensing, 6, 5696-5716. https://doi.org/10.3390/rs6065696

[21] Kamińska, A., Lisiewicz, M., Stereńczak, K., Kraszewski, B. and Sadkowski, R. (2018) Species-Related Single Dead Tree Detection Using Multi-Temporal ALS Data and CIR Imagery. Remote Sensing of Environment, 219, 31-43. https://doi.org/10.1016/j.rse.2018.10.005

[22] Ortiz, S.M., Breidenbach, J. and Kändler, G. (2013) Early Detection of Bark Beetle Green Attack Using TerraSAR-X and RapidEye Data. Remote Sensing, 5, 1912-1931. https://doi.org/10.3390/rs5041912

[23] Lausch, A., Heurich, M., Gordalla, D., Dobner, H.-J., Gwillym-Margianto, S. and 
Salbach, C. (2013) Forecasting Potential Bark Beetle Outbreaks Based on Spruce Forest Vitality Using Hyperspectral Remote-Sensing Techniques at Different Scales. Forest Ecology and Management, 308, 76-89. https://doi.org/10.1016/j.foreco.2013.07.043

[24] Waser, L.T., Küchler, M., Jütte, K. and Stampfer, T. (2014) Evaluating the Potential of WorldView-2 Data to Classify Tree Species and Different Levels of Ash Mortality. Remote Sensing, 6, 4515-4545. https://doi.org/10.3390/rs6054515

[25] Chua, R., Xie, Q. and Yuan, B. (2020) Crop Monitoring Using Multispectral Optical Satellite Imagery.

https://www.21at.sg/publication/publication/cotton-crop-monitoring-using-multisp ectral-optical-satellite-ima/

[26] Suet, Y.K., Min, E.N., Samuel, Y. and Bo, Y. (2017) TripleSat Imagery and Its Uses For Land Cover Analysis.

https://www.21at.sg/publication/publication/triplesat-imagery-and-its-uses-land-co ver-analysis/

[27] Belyaev, B.I., Stanchik, V.V., Krot, Yu.A., Weller, V.V., Domoratsky, A.V., Kazak, A.A. and Popkov, A.P. (2016) Mobile Photospectroradiometer and Two-Channel Modular Spectroradiometer for Field Measurements of Reflection Spectra of Objects. Proceedings of the 14th National Open Conference "Modern Problems of Remote Sensing of the Earth from Space”, Moscow, 14-18 November 2016, XIV.C.205. http://conf.rse.geosmis.ru/thesisshow.aspx?page $=0$ \&thesis $=5686$

[28] Katkovsky, L.V., Martinov, A.O., Krot, Y.A., Bruchkovskaya, S.I., Lomako, A.A., Silyuk, O.O., Stanchik, V.V. and Khomitsevich, A.D. (2016) Solar Spectropolarimeter SSP 600. Results of Ground-Based Measurements of Atmospheric Spectra. Proceedings of the 14th National Open Conference "Modern Problems of Remote Sensing of the Earth from Space”, Moscow, 14-18 November 2016, XIV.C.112. http://smiswww.iki.rssi.ru/d33 conf/thesisshow.aspx?page $=0$ \&thesis $=5593$

[29] Bruchkouskaya, S.I., Litvinovich, G.S., Bruchkousky, I.I. and Katkovsky, L.V. (2019) Algorithm for Second-Order Diffraction Correction in a Concave Diffraction Grating Spectrometer. Journal of Applied Spectroscopy, 86, 671-677.

https://doi.org/10.1007/s10812-019-00877-3

[30] L3Harris Geospatial (2020) TripleSat. https://www.13harrisgeospatial.com/Data-Imagery/Satellite-Imagery/High-Resoluti on/TripleSat

[31] Tsikman, I.M., Belyaev, Yu.V. and Popkov, A.P. (2012) Specialized Metrological Complex of Spectral-Energy Calibrations of Optical Remote Sensing Systems. Proceedings of the "Instrument-Making-2012" Conference, Minsk, 21-23 November 2012, 234-236.

[32] Krinov, Y.L. (1947) Spectral Reflectivity of Natural Formations. AN SSSR Press, Moscow. (In Russian)

[33] Index DataBase (2021) A Database for Remote Sensing Indices. https://www.indexdatabase.de/

[34] L3Harris Geospatial (2021) Alphabetical List of Spectral Indices https://www.l3harrisgeospatial.com/docs/alphabeticallistspectralindices.html

[35] Litvinovich, G.S., Silyuk, O.O., Bruchkovsky, I.I., Lomako, A.A., Belyaev, M.Yu. and Sarmin, E.E. (2020) Methodology for Determining Thematic Vegetation Indices for Solving the Problem of Searching for Drying Out Coniferous Stands, Proceedings of the 18th Russian National Open Conference "Modern Problems of Remote Sensing of the Earth from Space”, Moscow, 16-20 November 2020, XVIII.F.325. 
[36] Gaber, T., Tharwat, A., Ibrahim, A. and Hassanien, A.E. (2017) Linear Discriminant Analysis: A Detailed Tutorial. AI Communications, 30, 169-190. https://doi.org/10.3233/AIC-170729

[37] Bhaskaran, S., Devi, S., Bhatia, S., Samal, A. and Brown, L. (2013) Mapping Shadows in Very High-Resolution Satellite Data Using HSV and Edge Detection Techniques. Applied Geomatics, 5, 299-310. https://doi.org/10.1007/s12518-013-0118-4

[38] World Imagery Map Server (2020) ArcGIS Map Server. https://server.arcgisonline.com/ArcGIS/rest/services/World Imagery/MapServer/ 